\title{
Standardized Screening and Psycho-oncological Treatment of Orthopedic Cancer Patients
}

\author{
FLORIAN LENZE ${ }^{1}$, CHLODWIG KIRCHHOFF $^{2}$, FLORIAN POHLIG ${ }^{1}$, CAROLIN KNEBEL $^{1}$, HANS RECHL $^{1}$, \\ BIRGIT MARTEN-MITTAG ${ }^{3}$, PETER HERSCHBACH ${ }^{3,4}$, RÜDIGER V. EISENHART-ROTHE ${ }^{1}$ and ULRICH LENZE ${ }^{1}$ \\ Departments of ${ }^{1}$ Orthopedics and Sports Orthopedics, ${ }^{2}$ Trauma Surgery, \\ ${ }^{3}$ Department of Psychosomatic Medicine and Psychotherapy, ${ }^{4}$ Roman Herzog Comprehensive Cancer Center, \\ Klinikum rechts der Isar, Technical University of Munich, Munich, Germany
}

\begin{abstract}
Background/aim: Psycho-oncological distress is a relevant clinical problem. The aim of this study was to administer a standardized psycho-oncological distress screening for early identification and indication-based treatment of highly distressed orthopedic cancer patients as well as the evaluation of distress patterns. Patients and Methods: In total, 35 patients with cancer were psychooncologically screened using a cancer-specific expert rating scale (Basic Documentation for Psycho-Oncology Short Form) at three different time points (day of admission, day before discharge, 3 months postoperatively). Psychooncological support was offered to all patients whose distress exceeded a defined cut-off value. Results: Levels of distress in approximately $51 \%$ of patients exceeded the cutoff value at the time of admission and these patients received psycho-oncological support. The high distress levels decreased significantly over time. Patients whose distress did not exceed the cut-off value at the first assessment showed low distress levels at all time points. Conclusion: A relevant number of orthopedic tumor patients suffer from psychosocial distress. Standardized screening might help to identify and adequately treat those patients.
\end{abstract}

Approximately one-third of patients with cancer are reported to suffer from psychosocial distress, which constitutes a relevant problem both clinically and economically (1-3). Primary malignant musculoskeletal tumors are a rare and

This article is freely accessible online.

Correspondence to: Florian Lenze, MD, Department of Orthopedics and Orthopedic Sports Medicine, Klinikum rechts der Isar, Technical University Munich, Ismaninger Str. 22, 81675 Munich, Germany. E-mail: Flo_Lenze@yahoo.de

Key Words: Sarcoma, psychosocial distress, distress screening, cancer, bone tumor, PO-Bado. heterogeneous group of tumors constituting of approximately $1 \%$ of all malignant tumors (4). Nevertheless, patients with malignant musculoskeletal tumors are known to be particularly vulnerable to developing psychosocial difficulties (5-7). One explanation might be the fact that patients with musculoskeletal tumors are extremely affected by their diagnosis, since restricting and sometimes even mutilating operations (e.g. amputations) have to be performed in a relevant number of cases $(8,9)$. Patients with sarcoma are additionally often diagnosed at an early phase of life, with $60 \%$ being younger than 55 years of age (10).

Psychosocial distress is known to be associated with reduced quality of life and might additionally even be a predictor for a poor overall outcome in patients with cancer $(11,12)$. Studies on the psychosocial care of patients with cancer showed a lack of identification and adequate treatment for patients with psychosocial distress especially in early stages of the disease $(13,14)$. One clinical difficulty therein is that the somatic condition of a patient does not necessarily mirror the dimension of the actual distress level $(15,16)$. Moreover, about onequarter of such patients do not talk with their physicians about mental health problems unless invited to do so (17). Standardized psychosocial screening might therefore help to overcome this clinical problem. International guidelines suggest routine psychosocial distress screening for all patients with cancer, as well as provision of psycho-oncological support as needed (13). In patients with musculoskeletal tumors, however, standardized screening has not been implemented into cancer-specific treatment guidelines or treatment optimization studies in many countries (18).

Common instruments for identification of patients with cancer with psychosocial distress are self-rating questionnaires such as the Distress Thermometer (14), the Brief Symptom Inventory (15), or the Questionnaire on Distress in Cancer Patients (16). One of the major advantages of self-rating instruments is the ease of application, even in large numbers of patients, because no additional staff resources are required. Expert rating scales, 
in contrast, are usually based on standardized interviews and, in turn, offer some advantages compared with self-rating instruments. The Basic Documentation for Psycho-Oncology (PO-Bado) for instance, enables clinical staff to screen patients with cancer for the need for psycho-oncological support, as well as to assess psychosocial distress belonging to either the physical or psychological distress domain (19, 20). The aims of this study were to therefore: i) To establish a standardized psycho-oncological distress screening procedure at an orthopedic department; ii) to evaluate psychosocial distress patterns at different time points of the early treatment phase; and iii) to assess short-term effects of psycho-oncological treatment in those patients.

\section{Patients and Methods}

Thirty-five patients who were treated for a malignant musculoskeletal tumor at the Orthopedic Department of our University Hospital were prospectively enrolled. Inclusion criteria were: reliable diagnosis of a malignant (either primary or secondary) musculoskeletal tumor, requirement for a surgical procedure at our Orthopedic Department and an age of 18 years or above. Exclusion criteria were: benign musculoskeletal tumors, patients with pre-existing psychiatric disease/disorder and age below 18 years. All enrolled patients gave their informed consent to participate in this prospective study.

This study was approved by the local Ethics Committee (project number: 216/11) and registered as a clinical trial (NCT number: NCT01631175).

Procedures. At the time of elective admission to the Orthopedic Ward for surgery, all patients were asked to complete the 2-item depression screener Patient Health Questionnaire-2 (PHQ-2) of the Patient Health Questionnaire-9 (PHQ-9) to rule out a major depression disorder $(21,22)$. Patients with a total value of $\geq 3$ were considered ineligible for study inclusion and were excluded.

All enrolled participants were screened for psychosocial distress using the Basic Documentation for Psycho-Oncology Short Form (POBado SF) (23) at three defined time points (T1-T3): T1 (basic hospital assessment): within the course of inpatient admission to the orthopedic ward prior to surgery; T2 (repeat hospital assessment): after surgery on the day before discharge from the surgical ward; T3 (follow-up assessment): during clinic review, 3 months after the operation.

PO-Bado screening was performed at all time points by orthopedic doctors who were trained in conducting the standardized interview according to the published guidelines prior to the start of this study (23). Patients who exceeded the defined cut-off value of more than 9 points in the PO-Bado SF were assumed to be highly psycho-oncologically distressed and were offered psychosocial support. To this end, a first contact between agreeable patients and a staff member of the Department of Psychosomatic Medicine and Psychotherapy was made on the day of admission (day before surgery). Further treatment sessions were performed after surgery (during the hospital stay) depending on the individual demand of each patient. The psycho-oncological treatment included talking therapy as well as instructions for distress release strategies.

To evaluate perioperative distress patterns as well as to determine the 'acute-phase' effects of psychosocial support, a second PO-Bado
SF interview was performed on the day before discharge from the surgical ward (T2). The results of the psycho-oncological distress screening, as well as recommendations for the subsequent psychooncological outpatient treatment, were documented in the medical report and additionally presented within the Interdisciplinary Tumor Board meeting. The third screening (short-term effect) was conducted 3 months postoperatively (T3) during the standard outpatient follow-up appointment. In total, 28 participants $(80 \%)$ completed all three assessments, seven patients (20\%) were unavailable for the third assessment due to reduced overall condition, mortality, or loss to follow-up.

\section{Measures}

Patient Health Questionnaire 2 (PHQ-2). The PHQ-2 is a 2-item self-rating questionnaire, comprising the first two items of the PHQ9 questionnaire. The latter is a self-rating questionnaire, which, in contrast to other depression questionnaires, evaluates the presence of one out of the nine criteria of major depression of the Diagnostic and Statistical Manual of Mental Disorders - fourth edition (DSMIV) (21).

The PHQ-2 was validated as a screening tool for uncovering depression disorders $(21,22)$. For each of the two items, the response options are "not at all", "several days", "more than half the days" and "nearly every day", scored as $0,1,2$ and 3, respectively. A total score of 3 or more served as the cut-off value in this study (22) and was one of the exclusion criteria.

Sociodemographic and diagnosis-related data. In patients who did not meet the exclusion criteria and agreed to participate in the study, sociodemographic as well as medical data were collected prior to the psycho-oncological screenings (Table I). These were categorized into: i) Personal data: age, sex, relationship status, children, working situation; ii) tumor-related data: tumor type, metastasis, current state of disease, treatment during the past 2 months; and iii) general health-related data: further somatic disease and functional status [according to the World Health Organization - Eastern Cooperative Oncology Group (WHO-ECOG) performance scale (24)].

Basic documentation for PO-Bado SF (23). The PO-Bado SF is a cancer-specific screening instrument that enables clinical staff (including doctors and nurses) to screen patients with cancer for the need of psycho-oncological treatment based on a standardized interview of 5 to 10 minutes (20). The interview comprises three parts: questions on sociodemographic and medical characteristics (see above), as well as on the subjective experience of psychosocial distress. For the latter, the instrument refers to subjective experiences during the past 3 days. To this end, the PO-Bado SF consists of six items (Figure 1), which are rated on a scale ranging from 0 (not at all) to 4 (very much). Patients who exceeded the cutoff value of $>9$ points at any time point were considered to be highly psycho-oncologically distressed and assumed to require psychosocial support.

Statistical analysis. Data for sample description are presented as absolute and relative frequencies or means and standard deviations. For each PO-Bado SF item and for the total score, means and standard deviations were calculated. Mean differences between subgroups were analyzed using $t$-tests.

To investigate changes in total scores for PO-Bado SF over time and for different subgroups, repeated-measures analyses of variance 
Table I. Sociodemographic and diagnosis-related data $(n=35)$.

\begin{tabular}{|c|c|c|}
\hline Characteristic & & Value \\
\hline \multicolumn{3}{|l|}{ Personal data } \\
\hline Age (years) & Mean (SD) & $52.9(15)$ \\
\hline \multirow[t]{2}{*}{ Gender, n (\%) } & Male & $16(46)$ \\
\hline & Female & $19(54)$ \\
\hline \multirow[t]{2}{*}{ Steady relationship, n (\%) } & Yes & $28(80)$ \\
\hline & No & $7(20)$ \\
\hline \multirow[t]{2}{*}{ Children } & Yes & $20(57)$ \\
\hline & No & $15(43)$ \\
\hline \multirow[t]{5}{*}{ Working situation, n (\%) } & Employed & $12(34)$ \\
\hline & Home duties & $4(11)$ \\
\hline & Sick leave & $6(17)$ \\
\hline & Retired & $12(34)$ \\
\hline & Other & $1(3)$ \\
\hline \multicolumn{3}{|l|}{ Tumor-related data, n (\%) } \\
\hline \multirow[t]{4}{*}{ Tumor type } & Malignant soft-tissue tumor & $19(54)$ \\
\hline & Malignant bone tumor & $8(23)$ \\
\hline & Musculoskeletal metastasis of a solid tumor & $6(17)$ \\
\hline & Musculoskeletal manifestation of a hematological disease & $2(6)$ \\
\hline \multirow[t]{3}{*}{ Metastasis } & Yes & $12(34)$ \\
\hline & No & $18(51)$ \\
\hline & Not known & $5(14)$ \\
\hline \multirow[t]{3}{*}{ Current state of the disease } & First occurrence & $30(86)$ \\
\hline & Recurrence & $3(9)$ \\
\hline & Second tumor & $2(6)$ \\
\hline \multirow[t]{4}{*}{ Treatment during the past two months } & Surgery & $2(6)$ \\
\hline & Radiotherapy & $9(26)$ \\
\hline & Chemotherapy & $4(11)$ \\
\hline & None & $20(60)$ \\
\hline \multicolumn{3}{|l|}{ General health-related data, $\mathrm{n}(\%)$} \\
\hline \multirow[t]{2}{*}{ Further somatic disease } & Yes & $3(9)$ \\
\hline & No & $32(91)$ \\
\hline \multirow[t]{4}{*}{ Performance status (WHO-ECOG-Scale 0-4) } & 0 Normal activity & $20(57)$ \\
\hline & 1 Symptoms but nearly fully ambulatory & $5(14)$ \\
\hline & 2 Some bed time, but needs to be in bed less than $50 \%$ of normal walking hours & $7(20)$ \\
\hline & 3 Needs to be in bed more than $50 \%$ of normal walking hours & $3(9)$ \\
\hline
\end{tabular}

were performed with time as the within-subject factor and one of the following categories as a between-subjects factor: gender, metastases and psycho-oncological treatment (yes/no). All statistical tests were two-tailed and performed with SPSS version 22 (IBM Corp., Armonk, NY, USA). A p-value less than 0.05 was considered statistically significant.

\section{Results}

Sociodemographic and diagnosis-related data. The total sample comprised 35 patients. Personal data, tumor-related data and general health-related data are given in Table I.

\section{Psychological distress}

Basic hospital assessment (T1). The overall distress score of our patients $(\mathrm{n}=35)$ was 9.4 (range=1-19; $\mathrm{SD}=4.4)$. The mean scores for single items ranged from 0.94 to 2.23 , with a tendency for high distress in the psychological dimension. The highest mean (2.2) was found for "anxiety/worries" (Figure 2). At T1, a total of 18 patients $(51 \%)$ exceeded the cut-off score ( $>9$ points).

Categorical variables (gender, age, children, metastasis, tumor type, current state of disease, treatment during the previous 2 months, other somatic diseases and functional status) had no significant association with higher distress. There was a trend for higher distress in females $(p=0.058$; mean=10.6, $\mathrm{SD}=3.2$ ) and an association with the current state of the disease $(p=0.052$; mean $=16.0, \mathrm{SD}=4.2)$, but these did not reach statistical significance.

Psychosocial distress pattern (T1-T3). The mean distress level (of all included patients) at $\mathrm{T} 1, \mathrm{~T} 2$ and $\mathrm{T} 3$ was $9.4(\mathrm{SD}=4.3)$, 


\begin{tabular}{|c|c|c|c|c|c|}
\hline \multicolumn{6}{|c|}{ Please base your assessment on the subjective experience of the patient during the past three days } \\
\hline \multicolumn{6}{|c|}{ Psychosocial distress } \\
\hline The patient suffers from... & Not at all & A little & Moderate & Much & Very much \\
\hline ... Fatigue/tiredness & $\square_{0}$ & $\square_{1}$ & $\square_{2}$ & $\square_{3}$ & $\square_{4}$ \\
\hline ... Mood swings/uncertinity/helplessness & $\square$ & $\square$ & $\square$ & 口 & 口 \\
\hline ... Anxiety/worries/tension & $\square$ & $\square$ & $\square$ & $\square$ & 口 \\
\hline ... Grief/depression & $\square$ & 口 & $\square$ & 口 & ㅁ \\
\hline ... Functional limitations in daily livings & $\square$ & 口 & $\square$ & 口 & 口 \\
\hline ... Other problems, e.g. social or family problems & $\square$ & $\square$ & $\square$ & $\square$ & $\square$ \\
\hline \multicolumn{6}{|c|}{ Indication } \\
\hline & & & & Yes & No \\
\hline \multicolumn{4}{|c|}{ Currently, professional psycho-social support is indicated for the patient } & $\square$ & $\square$ \\
\hline
\end{tabular}

Figure 1. Basic documentation for Psycho-Oncology Short Form items.

$8.1(\mathrm{SD}=1.4)$ and $7.3(\mathrm{SD}=2.8)$, respectively. Comparison of distress levels at the different time points revealed a significant decrease of distress scores over time (T1-T3) $(p=0.032)$. Additionally, contrast analysis showed a statistically significant reduction in distress level from T1 to T2 $(p=0.09)$ and from $\mathrm{T} 1$ to $\mathrm{T} 3 \quad(p=0.018)$, whereas no significant difference was recorded between T2 and T3 ( $p=0.324)$.

Female patients showed significantly higher distress levels at all time points $(p=0.036)$ but no significant difference was seen in decrease over time when compared with male patients $(p=0.937)$ (Figure 3$)$. No significant influence of age, gender, relationship status, metastases and tumor type on change of psychosocial distress levels over time was detected.

Psychosocial distress over time with and without psychosocial treatment. In total, 18 patients exceeded the defined PO-Bado cut-off value ( $>9$ points) after the first assessment (basic hospital assessment), 15 of whom (83\%) agreed to receive psychosocial treatment during the hospital stay. First contact was established on the day of admission, and subsequent psychosocial consultation/treatment was conducted after a mean of 4 (range=2-6) days depending on the overall condition of the patient (operations on day 1 after admission).
The mean PO-Bado score for patients who did not exceed the cut-off value (and did not receive psycho-oncological treatment) was $5.5(\mathrm{SD}=2.5)$ at $\mathrm{T} 1,5.6(\mathrm{SD}=2.3)$ at $\mathrm{T} 2$ and 5.3 $(\mathrm{SD}=2.8)$ at $\mathrm{T} 3$, respectively. Patients whose distress did exceed the cut-off value and who received psycho-oncological treatment $(\mathrm{n}=15)$ had a mean PO-Bado score of $12.5(\mathrm{SD}=3.5)$ at $\mathrm{T} 1,9.7(\mathrm{SD}=2.0)$ at $\mathrm{T} 2$ and $9.0(\mathrm{SD}=3.6)$ at $\mathrm{T} 3$. While the distress level of patients who did not require psychooncological treatment was widely stable over all time points, in patients whose distress exceeded the cut-off value at $\mathrm{T} 1$, the distress level decreased markedly. This interaction effect was shown to be significant $(p=0.01)$. The distress level, however, was higher at any time point compared with the group who did not exceed the cut-off value at T1 (Figure 4).

\section{Discussion}

The aim of this study was to conduct a standardized psychooncological distress screening for early identification and subsequent indication-based psycho-oncological treatment of highly distressed orthopedic cancer patients. In our collective, more than one half of patients were highly distressed at the time of admission to the surgical ward and were assumed to require psychosocial support. The levels of patients whose distress exceeded the cut-off value and who 


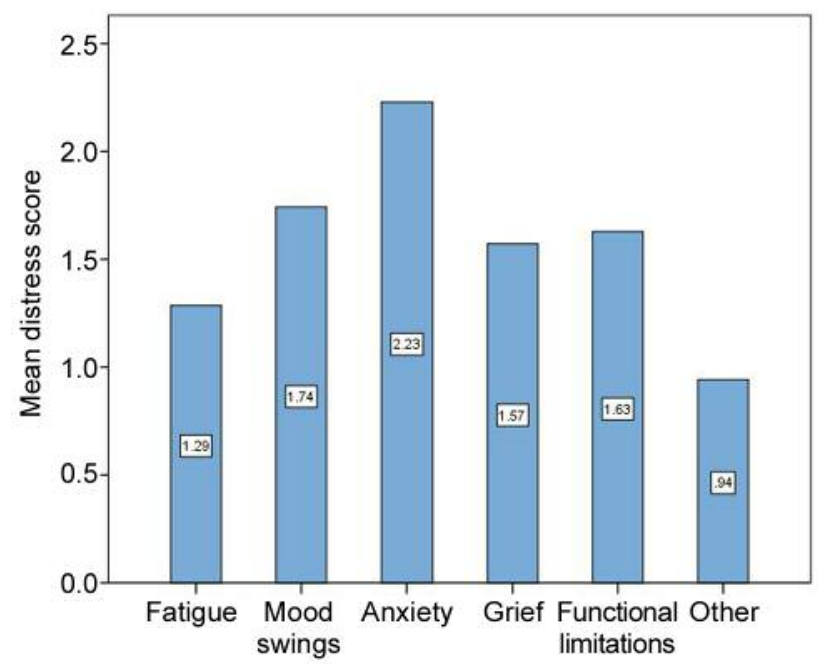

Figure 2. Mean scores for the single-items of the Basic Documentation for Psycho-Oncology at T1 (basic hospital assessment).

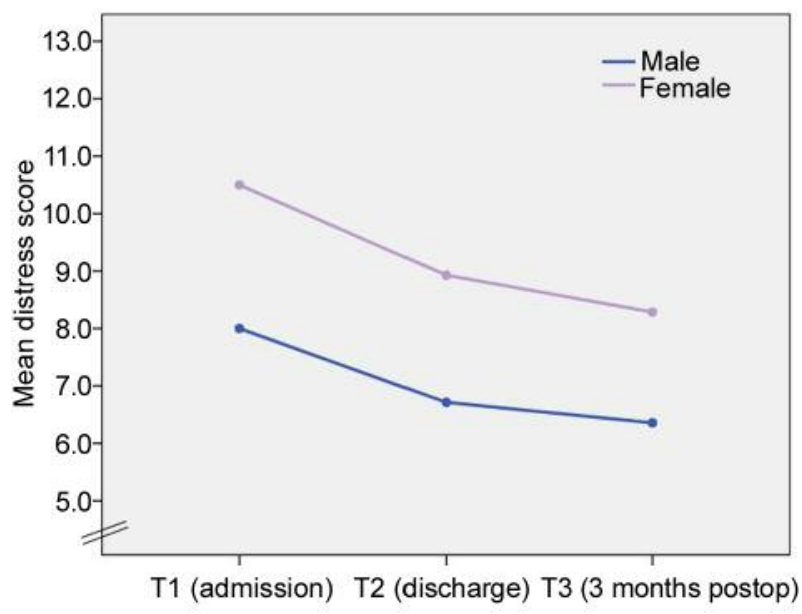

Figure 3. Distress scores for male and female participants over time (T1-T3).

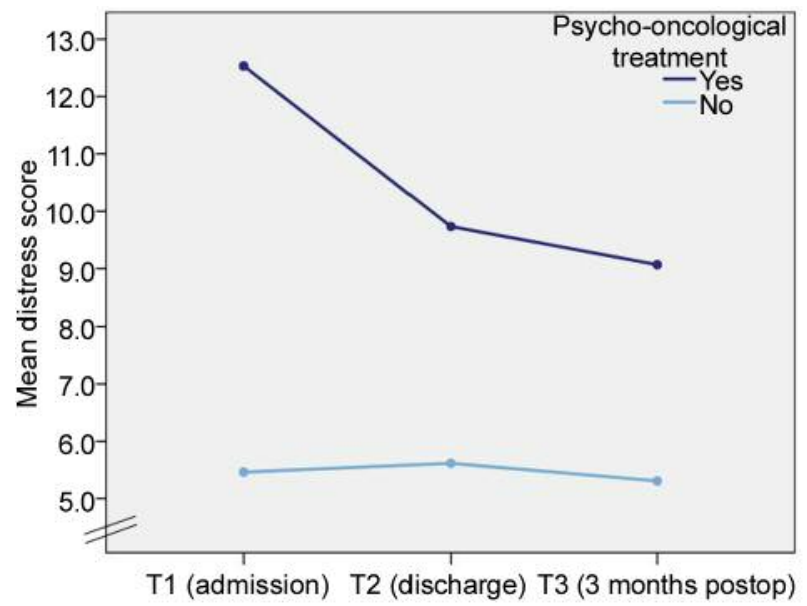

Figure 4. Distress scores of patients with and without psychooncological treatment (T1-T3). somewhat incomparable between different studies unless the same definitions are applied. Furthermore, different screening instruments (mainly self-rating questionnaires) are used in most studies, which mainly focus on general anxiety or depression but do not assess cancer-specific burdens in particular (17). Self-rating questionnaires additionally hold the risk of underestimating psychosocial distress levels, as some patients may tend to give socially desired answers rather than expressing their true mental health status.

Nevertheless, patients with orthopedic malignancies are known to be particularly vulnerable to psychosocial distress. In a study on psychological distress levels of 1,721 patients with different cancer entities, patients with soft-tissue sarcomas were found to have the highest distress levels (7). Distress levels in more than $40 \%$ of patients with soft-tissue sarcomas reached the cut-off value, indicating the need for psycho-oncological treatment (7). Recently, Pohlig et al. reported the results of their social-media-based cancerspecific online survey on distress levels in patients with osteosarcoma and Ewing's sarcoma (27). Distress in all of the included patients $(n=28)$ reached the defined cut-off value in their study. A potential selection bias, however, was acknowledged, as distressed patients are probably more likely to participate in patient communities of social networks (27).

The high number of patients with orthopedic tumors suffering from psychosocial distress might be attributed to the fear of stigmatization, even if unsubstantiated in most cases, life 
role changes or functional impairment. Accordingly, almost one half of the patients participating in our study were of working age and the majority reported highest levels of distress in the psychological dimension, such as anxiety. One major challenge is that only few patients with psychosocial distress are detected at an early phase of their disease, which might be explained by the low correlation between objective characteristics of the disease and the subjective perception of each patient $(13,16$, 27). Despite these difficulties, many clinicians do not routinely ask patients with cancer about psychosocial problems but rely on their own clinical judgement. In a study by Pirl et al., twothirds of oncologists reported that they screened patients for distress routinely, but fewer than $15 \%$ used a standardized screening instrument (28). Although frequently demanded for all patients with cancer, standardized psychosocial distress screening has not been integrated into various cancer-specific treatment guidelines and especially not for orthopedic cancer entities in many countries (18).

One aim of our study was, therefore, the establishment of a psycho-oncological screening algorithm (with screenings at different time points of the treatment) as well as an indicationbased psycho-oncological treatment of distressed orthopedic tumor patients at our institution. Screening at critical time points of the treatment, such as the time of entry into the cancer care system, has been found to be crucial for a successful implementation of psycho-oncological screening (29). In our study, the first psycho-oncological screening was therefore performed on the day of admission to allow for timely identification of distressed patients, as well as an early initiation of psycho-oncological treatment. Our results suggest that more than half of the patients are highly distressed at the time of admission, but a statistically significant reduction of distress levels was noted over the three defined time points. Contrast analysis revealed a significant reduction in the distress levels measured at the first time point compared with the second and third time points, respectively. Paredes et al. reported similar results in patients with sarcoma with moderate to large reduction of anxiety and depression symptoms between time of diagnosis and the follow-up phase (5). We hold the opinion, however, that high perioperative psycho-oncological distress levels as well as their reduction over time might partly be attributed to the operation itself, it being the most important part of active cancer treatment in such patients. Likewise, Tang et al. found in their prospective study on distress levels in patients with sarcoma of the extremity to be at the highest levels after tumor resection and to decrease after 6 and 12 months, respectively (25). Furthermore, distress was found to be associated with poorer outcome (e.g. quality of life) and poorer physical function (25). The effect of psycho-oncological treatment on the distress level of the enrolled patients was not investigated in their study however. In our study, the majority of patients (15/18) whose distress exceeded the defined cut-off value agreed to receive psycho-oncological treatment. The distress levels of those patients decreased significantly over time, whereas only 13 patients (11 had been distressed at T1, two had not been distressed at T1) had levels which exceeded the cut-off at T2 and only seven ( 1 ix had been distressed at $\mathrm{T} 1$ or $\mathrm{T} 2$, one had not been distressed at T1 or T2) at T3. Nevertheless, compared with patients whose distress did not exceed the cut-off value at T1, the distress level of those patients was still higher at all time points. As already mentioned above, whether this reduction was caused by active cancer treatment (tumor resection) or the shortterm effects of psychosocial treatment remains unclear. However, this was not the main focus of this study.

Our study has certain limitations. The study collective was somewhat small and heterogeneous with regard to tumor entities (primary and secondary malignant tumors, bone and soft-tissue tumors). No matched control group of distressed patients without the offer of psycho-oncological treatment was included for studying treatment effects more closely. Additionally, the follow-up period of 3 months was rather short and no conclusions can be drawn with regards to the mid-and long-term incidence of distress and effects of psycho-oncological treatment.

Musculoskeletal tumors, however, are particularly rare and the main emphasis of this study was the implementation of a psycho-oncological screening as well as an indicationbased treatment of distressed orthopedic cancer patients in general. We are of the opinion that psycho-oncological distress is a severe event and receiving proper treatment cannot be withheld from patients needing it.

\section{Conclusion}

A relevant number of orthopedic tumor patients suffer from psycho-oncological distress at the time of admission to the surgical ward prior to tumor resection. Our results underline the necessity for sufficient and standardized screening procedures for identification and treatment of highly distressed orthopedic tumor patients.

\section{Ethics Approval and Consent to Participate}

This study was approved by the local Ethics Committee (project number: 216/11). All enrolled patients gave their informed consent to participate in this study.

\section{Acknowledgements}

This study was supported by the Wilhelm-Sander Foundation (project number: 2009.905.2), which is a charitable, non-profit foundation for promotion of cancer research.

\section{Additional Information}

Information and instructions for the Basic Documentation for Psycho-Oncology Short Form (PO-Bado SF) are available at www.po-bado.med.tum.de. 


\section{References}

1 Book K, Marten-Mittag B, Henrich G, Dinkel A, Scheddel P, Sehlen S, Haimerl W, Schulte T, Britzelmeir I and Herschbach $\mathrm{P}$ : Distress screening in oncology-evaluation of the questionnaire on distress in cancer patients-short Form (Qsc-R10) in a German sample. Psychooncology 20(3): 287-293, 2011.

2 Dieng M, Cust AE, Kasparian NA, Mann GJ and Morton RL: Economic evaluations of psychosocial interventions in cancer: A systematic review. Psychooncology 25(12): 1380-1392, 2016.

3 Zabora J, BrintzenhofeSzoc K, Curbow B, Hooker C and Piantadosi S: The prevalence of psychological distress by cancer site. Psychooncology 10(1): 19-28, 2001.

4 Grimer RJ and Briggs TW: Earlier diagnosis of bone and softtissue tumours. J Bone Joint Surg Br 92(11): 1489-1492, 2010.

5 Paredes T, Canavarro MC and Simoes MR: Anxiety and depression in sarcoma patients: Emotional adjustment and its determinants in the different phases of disease. Eur J Oncol Nurs 15(1): 73-79, 2011.

6 Trautmann F, Singer S and Schmitt J: Patients with soft tissue sarcoma comprise a higher probability of comorbidities than cancer-free individuals. A secondary data analysis. Eur J Cancer Care (Engl) 26(6), 2017. doi: 10.1111/ecc.12605.

7 Herschbach P, Keller M, Knight L, Brandl T, Huber B, Henrich $\mathrm{G}$ and Marten-Mittag B: Psychological problems of cancer patients: A cancer distress screening with a cancer-specific questionnaire. Br J Cancer 91(3): 504-511, 2004.

8 Griesser MJ, Gillette B, Crist M, Pan X, Muscarella P, Scharschmidt $\mathrm{T}$ and Mayerson $\mathrm{J}$ : Internal and external hemipelvectomy or flail hip in patients with sarcomas: Qualityof-life and functional outcomes. Am J Phys Med Rehabil 91(1): 24-32, 2012.

9 Mason GE, Aung L, Gall S, Meyers PA, Butler R, Krug S, Kim M, Healey JH and Gorlick R: Quality of life following amputation or limb preservation in patients with lower extremity bone sarcoma. Front Oncol 3: 210, 2013.

10 Morrison BA: Soft tissue sarcomas of the extremities. Proc (Bayl Univ Med Cent) 16(3): 285-290, 2003.

11 Brown KW, Levy AR, Rosberger Z and Edgar L: Psychological distress and cancer survival: A follow-up 10 years after diagnosis. Psychosom Med 65(4): 636-643, 2003.

12 Frick E, Tyroller $M$ and Panzer M: Anxiety, depression and quality of life of cancer patients undergoing radiation therapy: A cross-sectional study in a community hospital outpatient centre. Eur J Cancer Care (Engl) 16(2): 130-136, 2007.

13 Carlson LE and Bultz BD: Cancer distress screening. Needs, models, and methods. J Psychosom Res 55(5): 403-409, 2003.

14 Mitchell AJ: Pooled results from 38 analyses of the accuracy of distress thermometer and other ultra-short methods of detecting cancer-related mood disorders. J Clin Oncol 25(29): 4670-4681, 2007.

15 Graves KD, Arnold SM, Love CL, Kirsh KL, Moore PG and Passik SD: Distress screening in a multidisciplinary lung cancer clinic: Prevalence and predictors of clinically significant distress. Lung Cancer 55(2): 215-224, 2007.

16 Siedentopf F, Marten-Mittag B, Utz-Billing I, Schoenegg W, Kentenich $\mathrm{H}$ and Dinkel A: Experiences with a specific screening instrument to identify psychosocial support needs in breast cancer patients. Eur J Obstet Gynecol Reprod Biol 148(2): 166-171, 2010.
17 Cape J and McCulloch Y: Patients' reasons for not presenting emotional problems in general practice consultations. Br J Gen Pract 49(448): 875-879, 1999.

18 Lenze U, Kirchhoff C, Book K, Pohlig F, Gradl G, Rechl H, Herschbach $\mathrm{P}$ and von Eisenhart-Rothe R: The role of psychooncology in orthopedic tumor centers. Orthopade 41(12): 958965, 2012.

19 Herschbach P, Book K, Brandl T, Keller M, Lindena G, Neuwohner K and Marten-Mittag B: Psychological distress in cancer patients assessed with an expert rating scale. Br J Cancer 99(1): 37-43, 2008.

20 Herschbach P, Book K, Brandl T, Keller M and Marten-Mittag $\mathrm{B}$ : The basic documentation for psycho-oncology (PO-BADO): An expert rating scale for the psychosocial experience of cancer patients. Onkologie 31(11): 591-596, 2008.

21 Kroenke K, Spitzer RL and Williams JB: The PHQ-9: Validity of a brief depression severity measure. J Gen Intern Med 16(9): 606-613, 2001

22 Kroenke K, Spitzer RL and Williams JB: The Patient Health Questionnaire-2: Validity of a two-item depression screener. Med Care 41(11): 1284-1292, 2003.

23 Marten-Mittag B, Book K, Buchhold B, Dinkel A, Grundobler B, Henrich G, Huber B, Pirker C, Regenberg A, Schickel S, Senf B, Wunsch A and Herschbach P: The basic documentation for Psycho-Oncology Short Form (PO-BADO SF)--an expert rating scale for distress screening: Development and psychometric properties. Psychooncology 24(6): 653-660, 2015.

24 Oken MM, Creech RH, Tormey DC, Horton J, Davis TE, McFadden ET and Carbone PP: Toxicity and response criteria of the Eastern Cooperative Oncology Group. Am J Clin Oncol 5(6): 649-655, 1982.

25 Tang MH, Castle DJ and Choong PF: Identifying the prevalence, trajectory, and determinants of psychological distress in extremity sarcoma. Sarcoma 2015: 745163, 2015.

26 Carlson LE, Angen M, Cullum J, Goodey E, Koopmans J, Lamont L, MacRae JH, Martin M, Pelletier G, Robinson J, Simpson JS, Speca M, Tillotson L and Bultz BD: High levels of untreated distress and fatigue in cancer patients. Br J Cancer 90(12): 2297-2304, 2004.

27 Pohlig F, Lenze U, Muhlhofer HML, Lenze FW, Schauwecker J, Knebel C, Zimmermann T and Herschbach P: IT-based psychosocial distress screening in patients with sarcoma and parental caregivers via disease-specific online social media communities. In Vivo 31(3): 443-450, 2017.

28 Pirl WF, Muriel A, Hwang V, Kornblith A, Greer J, Donelan K, Greenberg DB, Temel $\mathrm{J}$ and Schapira L: Screening for psychosocial distress: A national survey of oncologists. J Support Oncol 5(10): 499-504, 2007.

29 Bultz BD, Groff SL, Fitch M, Blais MC, Howes J, Levy K and Mayer C: Implementing screening for distress, the 6th vital sign: A Canadian strategy for changing practice. Psychooncology 20(5): 463-469, 2011.
Received May 6, 2018

Revised June 8, 2018

Accepted June 11, 2018 